2013

\title{
X-Ray And $\Gamma$-Ray Studies Of The Millisecond Pulsar And Possible X-Ray Binary/Radio Pulsar Transition Object Psr J1723-2837
}

Slavko Bogdanov

Paolo Esposito

Fronefield Crawford III

Andrea Possenti

Maura A. McLaughlin

See next page for additional authors

Follow this and additional works at: https://researchrepository.wvu.edu/faculty_publications

\section{Digital Commons Citation}

Bogdanov, Slavko; Esposito, Paolo; Crawford III, Fronefield; Possenti, Andrea; McLaughlin, Maura A.; and Freire, Paulo, "X-Ray And $\Gamma$-Ray Studies Of The Millisecond Pulsar And Possible X-Ray Binary/Radio Pulsar Transition Object Psr J1723-2837" (2013). Faculty Scholarship. 734.

https://researchrepository.wvu.edu/faculty_publications/734 
Authors

Slavko Bogdanov, Paolo Esposito, Fronefield Crawford III, Andrea Possenti, Maura A. McLaughlin, and Paulo Freire 


\title{
X-RAY AND $\gamma$-RAY STUDIES OF THE MILLISECOND PULSAR AND POSSIBLE X-RAY BINARY/RADIO PULSAR TRANSITION OBJECT PSR J1723-2837
}

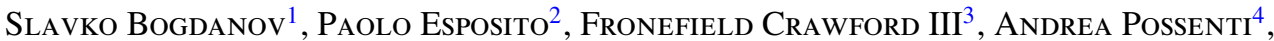 \\ Maura A. McLaughlin ${ }^{5}$, and Paulo Freire ${ }^{6}$ \\ ${ }^{1}$ Columbia Astrophysics Laboratory, Columbia University, 550 West 120th Street, New York, NY 10027, USA; slavko@ astro.columbia.edu \\ 2 INAF-IASF Milano, via East Bassini 15, I-20133 Milano, Italy \\ ${ }^{3}$ Department of Physics and Astronomy, Franklin and Marshall College, P.O. Box 3003, Lancaster, PA 17604, USA \\ ${ }^{4}$ INAF-Osservatorio Astronomico di Cagliari, Loc. Poggio dei Pini, Strada 54, I-09012 Capoterra (CA), Italy \\ ${ }^{5}$ Department of Physics and Astronomy, West Virginia University, 210E Hodges Hall, Morgantown, WV 26506, USA \\ ${ }^{6}$ Max-Planck-Institut für Radioastronomie, D-53121 Bonn, Germany \\ Received 2013 September 25; accepted 2013 November 22; published 2013 December 24
}

\begin{abstract}
We present X-ray observations of the "redback" eclipsing radio millisecond pulsar (MSP) and candidate radio pulsar/X-ray binary transition object PSR J1723-2837. The X-ray emission from the system is predominantly non-thermal and exhibits pronounced variability as a function of orbital phase, with a factor of $\sim 2$ reduction in brightness around superior conjunction. Such temporal behavior appears to be a defining characteristic of this variety of peculiar MSP binaries and is likely caused by a partial geometric occultation by the main-sequence-like companion of a shock within the binary. There is no indication of diffuse X-ray emission from a bow shock or pulsar wind nebula associated with the pulsar. We also report on a search for point source emission and $\gamma$-ray pulsations in Fermi Large Area Telescope data using a likelihood analysis and photon probability weighting. Although PSR J1723-2837 is consistent with being a $\gamma$-ray point source, due to the strong Galactic diffuse emission at its position a definitive association cannot be established. No statistically significant pulsations or modulation at the orbital period are detected. For a presumed detection, the implied $\gamma$-ray luminosity is $\lesssim 5 \%$ of its spin-down power. This indicates that PSR J1723-2837 is either one of the least efficient $\gamma$-ray producing MSPs or, if the detection is spurious, the $\gamma$-ray emission pattern is not directed toward us.
\end{abstract}

Key words: pulsars: general - pulsars: individual (PSR J1723-2837) - stars: neutron - X-rays: stars

Online-only material: color figures

\section{INTRODUCTION}

PSR J1723-2837 is a nearby ( $D=750 \mathrm{pc})$, binary, radio millisecond pulsar (MSP) with a $1.86 \mathrm{~ms}$ spin period discovered by Faulkner et al. (2004) in the Parkes Multibeam survey. Follow-up observations with the Parkes, Green Bank, and Lovell telescopes have allowed a positional localization to better than $1^{\prime \prime}$ and detailed parameterization of the binary orbit. Infrared, optical, and ultraviolet spectrophotometric studies have yielded complementary constraints on the properties of the companion star. The MSP follows an almost circular $14.8 \mathrm{hr}$ orbit, about a non-degenerate companion star of spectra type G5 with mass $0.4-0.7 M_{\odot}$ (Crawford et al. 2013). The pulsar is rarely detected at low frequencies, while it goes undetected at high radio frequencies for $\sim 15 \%$ of the orbit when the companion is generally closer to the Earth than the pulsar, suggesting that eclipses are responsible for the non-detections. This assertion is supported by the presence of peculiar orbital period irregularities in the radio timing residuals, which suggest strong tidal interactions between the neutron star and an extended and likely mass-losing companion. Occasionally, the pulsar goes undetected throughout the orbit (even when it is at the closest position with respect to the observer), indicating that, at times, the pulsar is completely enshrouded by matter released by its companion.

Its properties make PSR J1723-2837 a member of a growing class of eclipsing MSPs termed "redbacks," with low-mass main-sequence-like companions, found in both globular clusters and the field of the Galaxy, (e.g., D'Amico et al. 2001;
Archibald et al. 2009). The nature of their companions and the irregular eclipses and rapid dispersion measure fluctuations make them distinct from the so-called black widow eclipsing pulsars (Fruchter et al. 1988), which are bound to very low mass companions $\left(\lesssim 0.05 M_{\odot}\right)$. In this sense, PSR J1723-2837 appears similar to PSR J1023+0038, which is believed to still be transitioning from a low-mass X-ray binary (LMXB) to a fully "recycled" millisecond radio pulsar (Archibald et al. 2009; Patruno et al. 2013). The discovery of back-and-forth switching between accretion- and rotation-powered states of PSR J1824-2452I in the globular cluster M28 (Papitto et al. 2013) strongly supports the claim that redback systems are recently activated radio MSPs, but which may still sporadically revert to an accreting $\mathrm{X}$-ray binary state. At a distance $\sim 750 \mathrm{pc}$, based on its dispersion measure and the NE2001 model (Cordes \& Lazio 2002), and confirmed by optical spectroscopy (Crawford et al. 2013), PSR J1723-2837 is the nearest such system known, nearly a factor of two closer than PSR J1023+0038 ( $D=1.36 \mathrm{kpc}$; Deller et al. 2012). As such, it is a well-suited target for studies of various aspects of these peculiar systems.

In X-rays, redback systems typically exhibit predominantly non-thermal emission that is strongly modulated at the binary period (Bogdanov et al. 2005, 2010; Archibald et al. 2010; Bogdanov et al. 2011a, 2011b; Gentile et al. 2013). This radiation is likely produced by interaction of the energetic wind from the pulsar with material from the companion star. The large-amplitude flux variability likely arises due to a geometric occultation of the X-ray-emitting region by the secondary star (Bogdanov et al. 2011a). Studying this radiation can provide 
a valuable diagnostic of the physics and geometry of MSP winds, the interaction of the two stars, and collisionless shocks, in general.

The Fermi Large Area Telescope (LAT) has revealed that many MSPs are bright $\gamma$-ray sources, disproportionately accounting for 46 of the 132 pulsars detected in pulsed $\gamma$-rays ${ }^{7}$ (Abdo et al. 2013), including four of the six redbacks currently known (Hessels et al. 2011; Kaplan et al. 2012; Ray et al. 2012). As reported in Crawford et al. (2013), PSR J1723-2837 is not positionally coincident with a catalogued $\gamma$-ray source and no pulsations are detected with simple photon folding. Nevertheless, as recent studies have shown (Pletsch et al. 2012; Guillemot et al. 2012), in principle, it is still possible to detect pulsars in $\gamma$-rays by exploiting more sophisticated analysis techniques such as photon probability weighting.

Herein, we present XMM-Newton European Photon Imaging Camera (EPIC) and Chandra $X$-ray Observatory Advanced CCD Imaging Spectrometer (ACIS) observations of PSR J1723-2837. This study provides additional insight into the physics of this peculiar variety of binary MSPs and establishes the X-ray characteristics of this population. We also investigate the $\gamma$-ray emission from this pulsar based on the presently available Fermi-LAT data. The work is outlined as follows. In Section 2, we detail the observations, data reduction, and analysis procedures. In Section 3, we investigate the orbitalphase dependent variability of PSR J1723-2837 in X-rays. In Section 4 we present phase-averaged and phase-resolved X-ray spectroscopy, while in Section 5 we describe the X-ray imaging analysis. In Section 6 we attempt to constrain the physical properties of the intrabinary shock based on the X-ray data. In Section 7 we summarize the results of a Fermi-LAT analysis of the pulsar. We offer conclusions in Section 8.

\section{OBSERVATIONS AND DATA REDUCTION}

\subsection{XMM-Newton}

The XMM-Newton observation of PSR J1723-2837 (ObsID 0653830101) was conducted on 2011 March 3 for $55 \mathrm{ks}$. In this work, we concentrate on the data collected with the highthroughput EPIC instrument, which covers the $0.1-12 \mathrm{keV}$ band with one pn (Strüder et al. 2001) and two MOS (Turner et al. 2001) CCD cameras. All detectors used the medium-thickness optical blocking filters and were operated in Full Window mode.

The data were processed using version 11 of the XMMNewton Science Analysis Software $\left(\mathrm{SAS}^{8}\right)$ and standard screening criteria were applied (selecting only 1 and 2 pixel events using the default flag masks). The observation was affected by multiple intense soft proton flares. The corresponding periods of high particle background were excluded using intensity filters, following the method by De Luca \& Molendi (2004). This resulted in a net exposure time of $23.2 \mathrm{ks}$ in the pn, $31.7 \mathrm{ks}$ in the MOS 1, and $33.2 \mathrm{ks}$ in the MOS 2.

For the timing and spectral analysis, source events were extracted from each detector within a circular region with $40^{\prime \prime}$ radius, which contains $\sim 90 \%$ of the point source energy. The background counts were extracted from source-free regions on the same chip as the target. The ancillary response files and the spectral redistribution matrices were generated with the SAS

\footnotetext{
7 See https://confluence.slac.stanford.edu/display/GLAMCOG/Public+ List+of+LAT-Detected+Gamma-Ray+Pulsars for an up-to-date list. 8 The XMM-Newton SAS is developed and maintained by the Science Operations Centre at the European Space Astronomy Centre and the Survey Science Centre at the University of Leicester.
}

scripts arfgen and rmfgen, respectively. The spectra were binned so as to have a minimum of 30 counts per energy channel. For the variability analysis the data were barycentered using the DE405 ephemeris.

\subsection{Chandra}

The Chandra dataset was acquired on 2012 July 11 (ObsID 13713) in a continuous 49-ks effective exposure, covering 1.03 orbits of the pulsar. The radio pulsar position was at the aim point of the ACIS-S3 CCD set up in VFAINT mode and in a $1 / 8$ sub-array configuration to ensure that the effect of photon pileup (Davis 2001) is minimal. The re-processing, reduction, and analysis of the Chandra data were performed using $\mathrm{CIAO}^{9} 4.4$ (Fruscione et al. 2006) and the corresponding calibration products (CALDB 4.4.10). To facilitate the identification of nebular X-ray emission, we reprocessed the level 1 data products opting for no pixel randomization and used the background cleaning procedure appropriate for the VFAINT mode. However, since this algorithm can reject genuine source photons for relatively bright sources, no background cleaning was applied to the data used for the spectroscopy and variability studies.

For the purposes of the investigations presented in Sections 3 and 4 , we extracted photons within $2^{\prime \prime}$ of the source. To permit spectral fitting, the source counts in the $0.3-8 \mathrm{keV}$ band were combined such that at least 15 counts per energy bin were obtained. The background spectrum was obtained from regions near the pulsar that are devoid of point sources. For the purposes of the variability study, the event times of arrival were shifted to the solar system barycenter assuming DE405 JPL solar system ephemeris.

The spectroscopic analyses of both the XMM-Newton and Chandra observations were carried out using XSPEC ${ }^{10}$ 12.7.1. The coarse time resolution of the observations $(0.4 \mathrm{~s}$ for Chandra/ACIS-S, $2.6 \mathrm{~s}$ and $0.73 \mathrm{~s}$ for XMM-Newton/EPIC MOS and pn, respectively) does not permit a search for pulsations at the pulsar spin period.

\subsection{Fermi-LAT}

For the $\gamma$-ray analysis, we retrieved Pass7 Fermi-LAT event data from 2008 August 4 and 2013 June 11 within $20^{\circ}$ of the pulsar position and accompanying spacecraft data. The analysis was carried out using the Fermi Science Tools ${ }^{11}$ v9r27p1. Following the recommended guidelines from the Fermi Science Support Center, the data were filtered for "source" class events in good time intervals with energies above $100 \mathrm{MeV}$, zenith angles smaller than $100^{\circ}$, and telescope rocking angles $\leqslant 52^{\circ}$ using the gtselect and gtmktime tools. The spatial/spectral binned likelihood analysis was conducted using the gtlike tool based on the input counts, exposure, and source maps, lifetime cube and source model generated with the Fermi Science Tools.

Using the spectral parameters obtained from the likelihood analysis, the tool gtsrcprob was used to assign each event a probability that it originated from PSR J1723-2837 based on the fluxes and spectra obtained from the likelihood analysis. The gtdiffrsp was used to compute the integral over solid angle of a diffuse source model convolved with the instrumental response function. Only photons with probabilities $\geqslant 0.05$ of being associated with the pulsar were folded. To fold the data

\footnotetext{
9 Chandra Interactive Analysis of Observations, available at http://cxc.harvard.edu/ciao/.

10 Available at http://heasarc.nasa.gov/docs/xanadu/xspec/index.html.

11 http://fermi.gsfc.nasa.gov/ssc/data/analysis/scitools/overview.html
} 


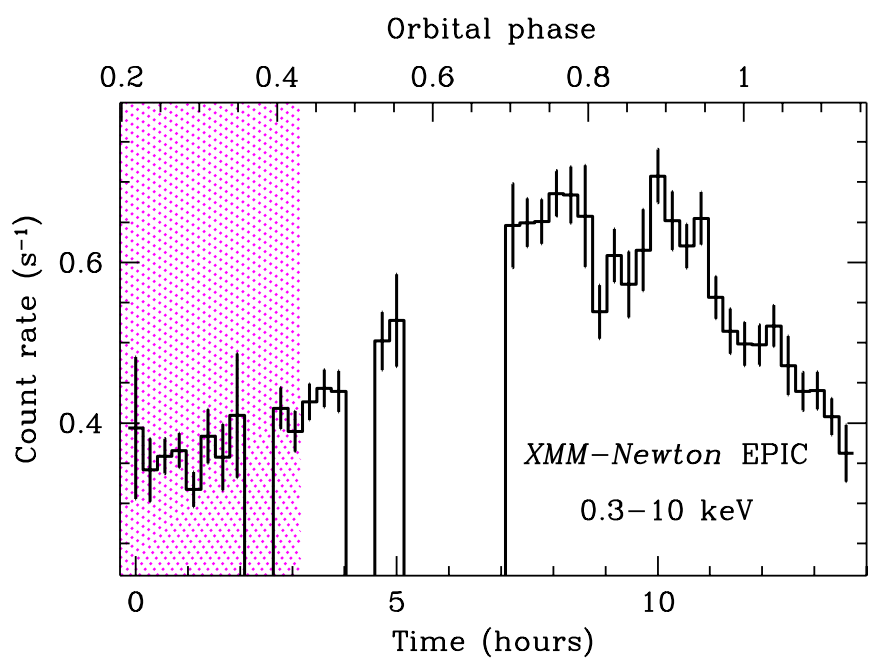

Figure 1. Background-subtracted $X M M-N e w t o n / E P I C$ lightcurve of PSR $\mathrm{J} 1723-2837$ in the $0.3-10 \mathrm{keV}$ band binned in $1 \mathrm{ks}$ intervals. The gaps in the data are intervals that have been removed due to excessive background flaring. The dotted magenta band represents the approximate portion of the orbit where the pulsar undergoes a radio eclipse at $2 \mathrm{GHz}$. The orbital phase is defined such that the companion star is between the pulsar and observer at $\phi_{b}=0.25$. (A color version of this figure is available in the online journal.)

we used the fermi plugin for tempo2 and the radio ephemeris presented in Crawford et al. (2013).

\section{X-RAY ORBITAL VARIABILITY}

Using the radio timing ephemeris of PSR J1723-2837 (Crawford et al. 2013) we have determined the orbital phases of the barycentered XMM-Newton and Chandra source photons. Each observation cover approximately a single orbit. Largeamplitude flux variability as a function of time is clearly evident in both data sets (Figures 1 and 2). Although substantial segments of the XMM-Newton data are removed due to strong flaring, the trend in the flux modulation is still apparent. A substantial decrease (by a factor of $\sim 2-3$ ) in flux at superior conjunction $\left(\phi_{b} \approx 0.25\right)$ is apparent. This indicates that the X-ray flux varies as a function of the binary period (Figures 1 and 2), a behavior similar to what is observed analogous MSP systems, especially PSRs J1023+0038 (Archibald et al. 2010; Bogdanov et al. 2011a) in the field of the Galaxy and J0024-7204W in the globular cluster 47 Tuc (Camilo et al. 2000; Freire et al. 2003; Bogdanov et al. 2005).

A $\chi^{2}$ test on the data, binned as in Figures 1 and 2, indicates negligible probabilities of $4 \times 10^{-94}(20.5 \sigma)$ and $2 \times 10^{-14}(7.6 \sigma)$ that the observed flux variability arises from a constant flux distribution for XMM-Newton and Chandra, respectively. A more robust estimate is obtained from the Kuiper test (Paltani 2004), which considers the unbinned lightcurves, weighted to account for the non-uniform exposure across the orbit. This approach gives probabilities of $6 \times 10^{-101}(21.3 \sigma)$ and $2.8 \times 10^{-21}(9.4 \sigma)$, for the XMM-Newton and Chandra data respectively, that events being drawn from a constant distribution would exhibit this level of non-uniformity. There is no appreciable spectral variability as a function of orbital phase (bottom panel of Figure 2). There is only marginally significant evidence for spectral hardening between phases $\sim 0.7-0.9$, although as discussed below, pile-up in the Chandra data may be partly responsible for this.

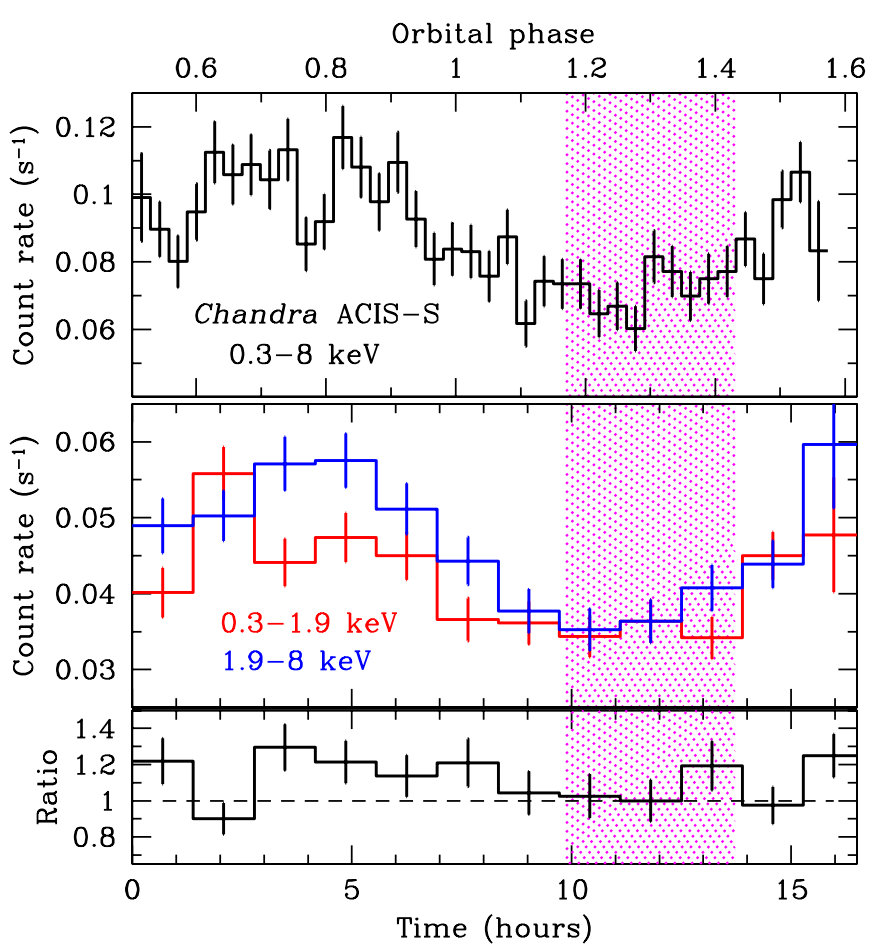

Figure 2. Chandra/ACIS-S $0.3-8 \mathrm{keV}$ (top panel) and $0.3-1.9 \mathrm{keV}$ and $1.9-8 \mathrm{keV}$ bands (middle panel) lightcurves of PSR J1723-2837 vs. time and orbital phase. The background contributes negligibly to the total count rate $(\sim 0.1 \%)$. Bottom panel: the ratio obtained by dividing the $1.9-8 \mathrm{keV}$ by the 0.3-1.9 keV lightcurve. The radio eclipse interval at $2 \mathrm{GHz}$ is shown by the magenta dotted band.

(A color version of this figure is available in the online journal.)

\section{PHASE-AVERAGED X-RAY SPECTROSCOPY}

In similar MSP binary systems, the phase-integrated X-ray continuum is well represented by a power-law, while a single thermal (either blackbody or neutron star hydrogen atmosphere) model fails to reproduce the spectral shape. In some instances, an acceptable fit is obtained with a composite power-law plus thermal model. Like many MSPs studied in X-rays (Zavlin 2006; Bogdanov et al. 2006; Bogdanov \& Grindlay 2009), PSR J1723-2837 is expected to have hot polar caps due to a return current of particles from the pulsar magnetosphere. Based on this, in addition to a single-component power-law we consider a composite model consisting of an absorbed power-law and a neutron star atmosphere. We choose the NSA atmosphere model (Zavlin et al. 1996) instead of a blackbody since it has been shown that the thermal pulsations from the nearest MSPs favor an atmosphere (Zavlin \& Pavlov 1998; Bogdanov et al. 2007; Bogdanov \& Grindlay 2009; Bogdanov 2013), as expected at the surface of objects "recycled" via accretion of matter. A fraction of the X-rays associated with J1723-2837 could arise from a thermal plasma within or around the binary, possibly from the active corona of the companion star or intrabinary plasma that causes the radio eclipses. Hence, we also present fits using a power-law plus MEKAL hot diffuse plasma model, which includes line emissions from several elements based on input metal abundances (Mewe et al. 1985, 1986; Liedahl et al. 1995). Table 1 summarizes the results using the three different models.

A joint fit to the XMM-Newton and Chandra phase-averaged spectra results in statistically unacceptable fits due to a significant flux difference between the two data sets. This discrepancy 
Table 1

Summary of X-Ray Spectroscopy for PSR J1723-2839

\begin{tabular}{|c|c|c|c|c|c|c|}
\hline \multirow[t]{2}{*}{ Model $^{\mathrm{a}}$} & \multirow{2}{*}{$\begin{array}{c}\boldsymbol{X} \boldsymbol{M} \boldsymbol{M} \\
\text { Total }\end{array}$} & \multirow{2}{*}{$\begin{array}{c}\boldsymbol{X} \boldsymbol{M}+\boldsymbol{C} \boldsymbol{X} \boldsymbol{O} \\
\text { Total }\end{array}$} & \multicolumn{4}{|c|}{$C X O$} \\
\hline & & & Total & $\begin{array}{c}\phi_{b, 1} \\
(0.0-0.5)\end{array}$ & $\begin{array}{c}\phi_{b, 2} \\
(0.5-1.0)\end{array}$ & $\begin{array}{c}\text { Joint } \\
\phi_{b, 1}+\phi_{b, 2}\end{array}$ \\
\hline \multicolumn{7}{|c|}{ Power-law } \\
\hline$N_{\mathrm{H}}\left(10^{21} \mathrm{~cm}^{-2}\right)$ & $2.0_{-0.09}^{+0.09}$ & $1.9_{-0.08}^{+0.08}$ & $1.6_{-0.2}^{+0.2}$ & $1.8_{-0.3}^{+0.4}$ & $1.3_{-0.2}^{+0.3}$ & $1.5_{-0.2}^{+0.2}$ \\
\hline$\Gamma$ & $1.15_{-0.02}^{+0.02}$ & $1.13_{-0.02}^{+0.02}$ & $1.00_{-0.04}^{+0.04}$ & $1.10_{-0.07}^{+0.07}$ & $0.88_{-0.06}^{+0.06}$ & $1.13_{-0.05}^{+0.05} / 0.85_{-0.04}^{+0.05}$ \\
\hline$F_{X}(0.3-8 \mathrm{keV})^{\mathrm{c}}$ & $1.87_{-0.02}^{+0.02}$ & $1.87_{-0.02}^{+0.02} / 1.27_{-0.02}^{+0.02}$ & $1.29_{-0.02}^{+0.02}$ & $1.06_{-0.03}^{+0.03}$ & $1.48_{-0.04}^{+0.04}$ & $1.05_{-0.03}^{+0.03} / 1.49_{-0.04}^{+0.03}$ \\
\hline$\chi_{v}^{2} /$ dof & $1.04 / 321$ & $1.02 / 519$ & $0.97 / 199$ & $1.00 / 94$ & $0.95 / 129$ & $0.98 / 225$ \\
\hline \multicolumn{7}{|c|}{ Power-law + NSA ${ }^{b}$} \\
\hline$N_{\mathrm{H}}\left(10^{21} \mathrm{~cm}^{-2}\right)$ & $3.4_{-0.8}^{+0.7}$ & $2.1_{-0.2}^{+0.3}$ & $0.96_{-0.93}^{+0.42}$ & $5.0_{-1.6}^{+1.1}$ & $4.0_{-2.2}^{+1.3}$ & $0.80_{-0.72}^{+0.58}$ \\
\hline$\Gamma$ & $1.15_{-0.04}^{+0.04}$ & $1.12_{-0.02}^{+0.02}$ & $0.77_{-0.68}^{+0.26}$ & $1.33_{-0.12}^{+0.10}$ & $0.97_{-0.11}^{+0.10}$ & $0.82_{-0.54}^{+0.22}$ \\
\hline$T_{\text {eff }}\left(10^{6} \mathrm{~K}\right)$ & $0.82_{-0.17}^{+0.30}$ & $1.10_{-0.27}^{+0.75}$ & $<6.59$ & $0.32_{-0.41}^{+0.27}$ & $0.60_{-0.13}^{+0.74}$ & $0.50_{-0.08}^{+0.22}$ \\
\hline$R_{\mathrm{eff}}(\mathrm{km})$ & $4.3_{-4.1}^{+9.9}$ & $<0.32$ & $<0.05$ & $115_{-112}^{+201}$ & $11.6_{-11.5}^{+31.3}$ & $14.5_{-14.2}^{+30.3}$ \\
\hline Non-thermal fraction ${ }^{\mathrm{d}}$ & $0.99_{-0.04}^{+0.01}$ & $0.98_{-0.12}^{+0.02} / 0.97_{-0.12}^{+0.3}$ & $0.93_{-0.03}^{+0.03}$ & $0.41_{-0.08}^{+0.06}$ & $0.84_{-0.04}^{+0.05}$ & $0.84_{-0.07}^{+0.08} / 0.89_{-0.06}^{+0.07}$ \\
\hline$F_{X}(0.3-8 \mathrm{keV})^{\mathrm{c}}$ & $2.23_{-0.03}^{+0.04}$ & $1.91_{-0.03}^{+0.04} / 1.29_{-0.03}^{+0.03}$ & $1.41_{-0.07}^{+0.05}$ & $2.90_{-0.04}^{+0.04}$ & $1.82_{-0.07}^{+0.05}$ & $1.06_{-0.07}^{+0.06} / 1.49_{-0.06}^{+0.06}$ \\
\hline$\underline{\chi_{v}^{2} / \text { dof }}$ & $1.01 / 316$ & $1.03 / 517$ & $0.98 / 197$ & $0.98 / 92$ & $0.95 / 127$ & $0.98 / 222$ \\
\hline \multicolumn{7}{|c|}{ Power-law + MEKAL } \\
\hline$N_{\mathrm{H}}\left(10^{21} \mathrm{~cm}^{-2}\right)$ & $2.1_{-0.1}^{+0.2}$ & $2.0_{-0.1}^{+0.2}$ & $2.2_{-0.5}^{+0.6}$ & $3.6_{-1.2}^{+1.4}$ & $1.3_{-0.3}^{+0.3}$ & $0.19_{-0.5}^{+0.6}$ \\
\hline$\Gamma$ & $1.14_{-0.02}^{+0.03}$ & $1.13_{-0.02}^{+0.02}$ & $1.05_{-0.06}^{+0.06}$ & $1.23_{-0.11}^{+0.11}$ & $0.85_{-0.07}^{+0.07}$ & $1.08_{-0.07}^{+0.07} / 0.95_{-0.07}^{+0.07}$ \\
\hline$k T(\mathrm{keV})$ & $0.57_{-0.27}^{+0.14}$ & $0.32_{-0.06}^{+0.12}$ & $0.25_{-0.05}^{+0.13}$ & $0.24_{-0.03}^{+0.06}$ & $<1.41$ & $0.29_{-0.08}^{+0.19}$ \\
\hline Non-thermal fraction ${ }^{\mathrm{d}}$ & $0.99_{-0.04}^{+0.01}$ & $0.99_{-0.03}^{+0.01} / 0.98_{-0.03}^{+0.02}$ & $0.97_{-0.03}^{+0.03}$ & $0.88_{-0.08}^{+0.06}$ & $0.99_{-0.06}^{+0.01}$ & $0.98_{-0.12}^{+0.02} / 0.98_{-0.12}^{+0.02}$ \\
\hline$F_{X}(0.3-8 \mathrm{keV})^{\mathrm{c}}$ & $1.89_{-0.02}^{+0.02}$ & $1.91_{-0.03}^{+0.04} / 1.29_{-0.03}^{+0.03}$ & $1.34_{-0.02}^{+0.11}$ & $1.29_{-0.05}^{+0.06}$ & $1.49_{-0.04}^{+0.04}$ & $1.09_{-0.07}^{+0.07} / 1.53_{-0.06}^{+0.06}$ \\
\hline$\chi_{v}^{2} /$ dof & $1.03 / 316$ & $1.02 / 517$ & $0.97 / 197$ & $0.99 / 92$ & $0.95 / 127$ & $0.98 / 222$ \\
\hline
\end{tabular}

Notes.

a All quoted uncertainties and limits are at a $1 \sigma$ confidence level.

${ }^{\mathrm{b}}$ For the nsa model, a star with $R=10 \mathrm{~km}, M=1.4 M_{\odot}$ is assumed. The un-redshifted effective radius, $R_{\mathrm{eff}}$, was computed assuming $D=750$ pc.

${ }^{c}$ Unabsorbed X-ray flux $(0.3-8 \mathrm{keV})$ in units of $10^{-12} \mathrm{erg} \mathrm{cm}^{-2} \mathrm{~s}^{-1}$.

${ }^{\mathrm{d}}$ Fraction of unabsorbed flux from the non-thermal component in the $0.3-8 \mathrm{keV}$ band.

e For the MEKAL model, solar abundances are assumed.

cannot be explained by the partial orbital coverage of the XMMNewton data. Given the $1.3 \mathrm{yr}$ separation of the two observations, it is likely the result of long term flux variations from the $X$-ray-emitting intrabinary shock, possibly due to changes in the stellar activity of the secondary star or fluctuations in the outflow of gas from the companion through the inner Lagrangian point. Similar flux changes on timescales of years have also been observed in the "redback" PSR J0024-7204W in the globular cluster 47 Tuc (Bogdanov et al. 2005; Cameron et al. 2007), suggesting this may be a common feature of these systems. As a consequence of the appreciable flux difference, we have fitted the two observations jointly but with independent flux normalizations as well as separately (Figure 3). From a statistical standpoint, the simple pure power-law and power-law plus thermal component both produce satisfactory fits. The addition of a NSA or MEKAL component results in a slight improvement in the quality of the fit compared to a pure power-law but is not statistically significant. The parameters of the NSA and MEKAL components are poorly constrained as their contribution to the total flux is typically only a few percent. The hydrogen column density through the Galaxy along the line of sight to the pulsar is $\sim 3.8 \times 10^{21} \mathrm{~cm}^{-2}$ based on Kalberla et al. (2005). The best-fit values of $N_{\mathrm{H}}$ (assuming abundances from Anders \& Grevesse 1989) in Table 1 are generally consistent with this value.

\subsection{Orbital Phase-resolved Spectrum}

In the case of the XMM-Newton data, the large gaps in orbital phase coverage resulting from background flare removal are not conducive to phase-resolved spectroscopy. Therefore, we only consider the Chandra observation for this analysis. We divide the data over two orbital phase intervals: $\phi_{b}=0.0-0.5$ (around the minimum in X-ray flux) and $\phi_{b}=0.5-1.0$. The two spectra were fitted both separately and jointly. For the latter, for the thermal component we tied both the temperature and effective radius in all instances since the emission associated with the pulsar is not likely to exhibit any variability as a function of orbital phase. The best-fit parameters of the orbital phaseresolved spectral fits are listed in of Table 1.

A simple power-law provides a very good fit to the total phaseaveraged X-ray spectrum, as well as each of the spectra at phases $0-0.5$ and $0.5-1$. The same is true for the joint fit of phaseresolved spectra. In all instances, the power-law around flux maximum is significantly harder compared to flux minimum and inferred from the phase-averaged XMM-Newton data. Although this could be indicative of intrinsic spectral hardening of the source, it could also arise due to photon pile-up on the ACIS-S detector. Pile-up occurs when two or more photons, arriving at the detector during one frame time, are erroneously identified as a single photon with the sum of the photon energies or else 


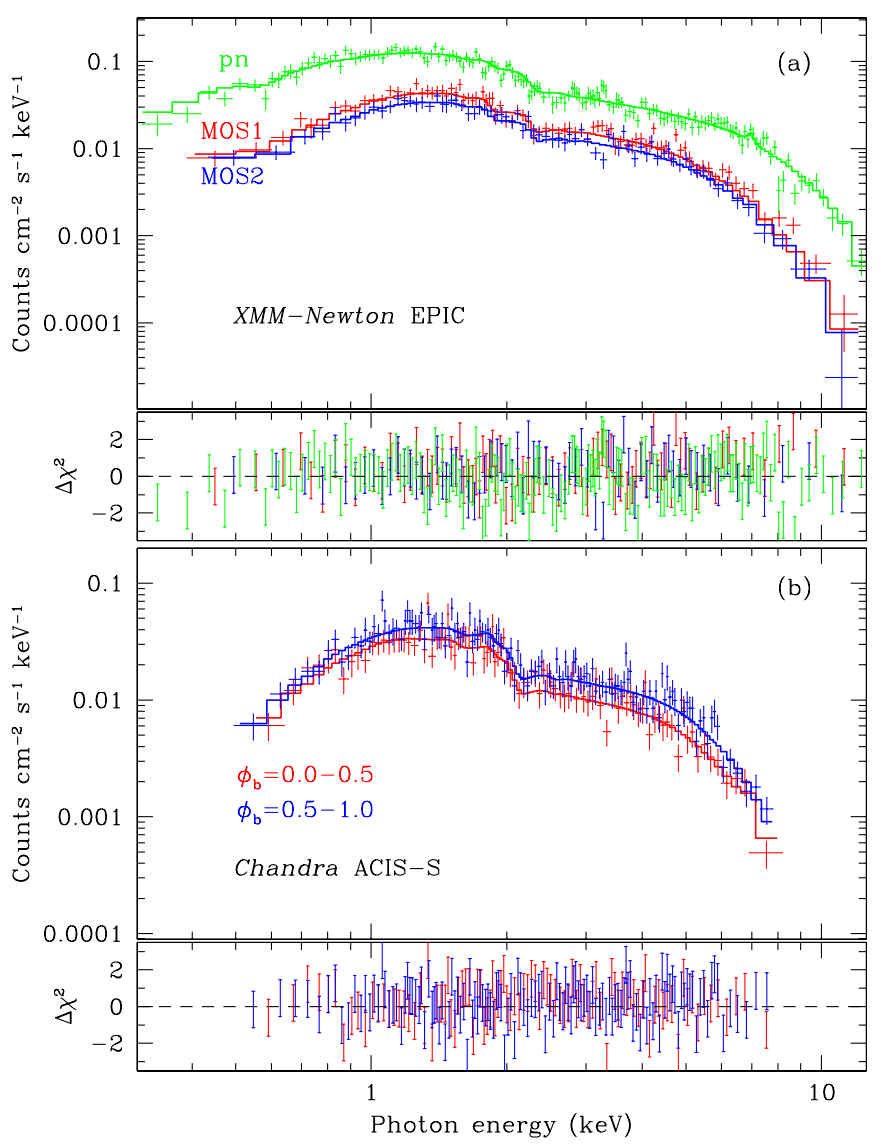

Figure 3. (a) XMM-Newton/EPIC MOS1/2 (red and blue) and pn (green) orbital phase-averaged X-ray spectra fitted with an absorbed power-law. (b) Orbital phase-resolved Chandra X-ray spectral continua of PSR J1723-2837 for phases $\phi_{b}=0.0-0.5$ (blue) and $\phi_{b}=0.5-1.0$ (red), fitted with an absorbed power-law. The lower panels shows the best-fit residuals in terms of $\sigma$ with error bars of size one. See text and Table 1 for best-fit parameters.

(A color version of this figure is available in the online journal.)

discarded (Davis 2001). The result is a distortion of the intrinsic shape of the source spectrum, causing an artificial hardening of the spectrum. Using PIMMS, ${ }^{12}$ we find that the predicted pile-up for this source in the $1 / 8$ sub-array mode of ACIS-S is $\sim 2 \%$ based on the phase-averaged count rate. Indeed, fitting a power-law spectrum with the pileup model in XSPEC yields a slightly steeper power-law with $\Gamma=1.05_{-0.07}^{+0.05}$ for the phaseaveraged ACIS-S spectrum and $\Gamma=0.94_{-0.05}^{+0.07}$ for the orbital phase interval $\phi_{b}=0.5-1.0$, which is more consistent with the results from the other fits.

\section{X-RAY IMAGING ANALYSIS}

PSR J1723-2837 is by far the brightest source in the XMM-Newton/EPIC and Chandra/ACIS-S images (Figure 4). This confirms that the ROSAT X-ray point source $1 \mathrm{RXS}$ $\mathrm{J} 172323.7-283805$, located $13^{\prime \prime}$ from the position of the pulsar, is in fact PSR J1723-2837, as suggested in Crawford et al. (2013). The X-ray position measured from the Chandra image using wavdetect is $\alpha_{X}=17^{\mathrm{h}} 23^{\mathrm{m}} 23^{\mathrm{s}} .19, \delta_{X}=-28^{\circ} 37^{\prime} 57^{\prime \prime} .49$, which differs from the radio position $\alpha_{r}=17^{\mathrm{h}} 23^{\mathrm{m}} 23^{\mathrm{s}} .1856$, $\delta_{r}=+17^{\circ} 37^{\prime} 57^{\prime \prime} .17$ (Crawford et al. 2013) by just $+0^{\prime \prime} .06$ and -0.32 in right ascension and declination, respectively,

$\overline{12}$ Available at http://cxc.harvard.edu/toolkit/pimms.jsp.

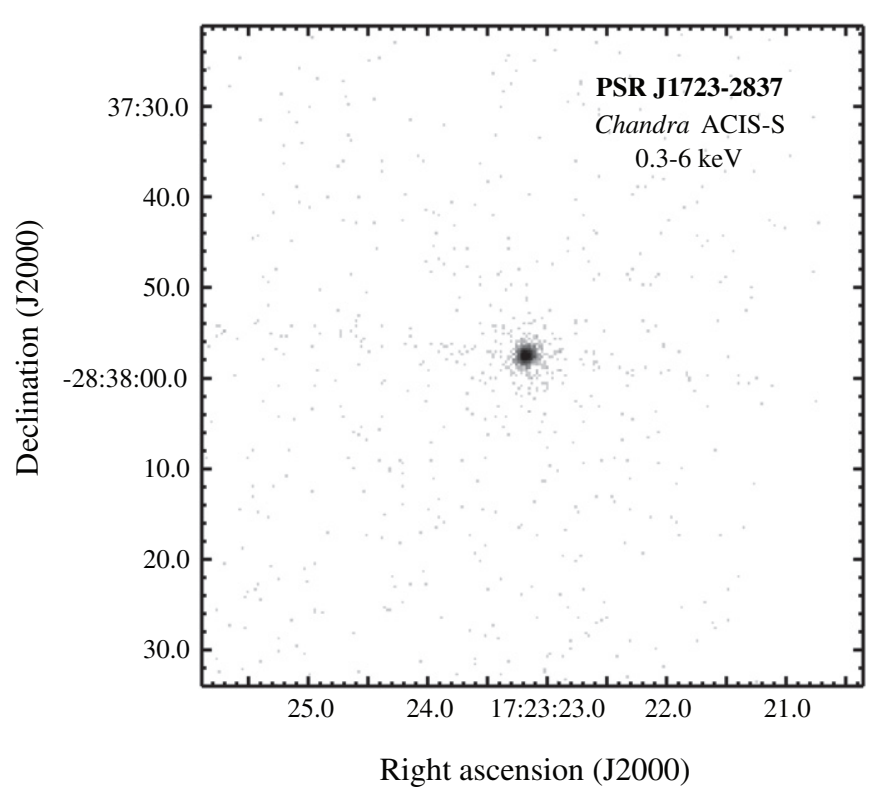

Figure 4. Chandra/ACIS-S3 J2000 1'.2 × 1'.2 image binned at the intrinsic resolution of the ACIS-S detector (0.5) showing the X-ray counterpart to PSR J1723-2837 in the $0.3-6 \mathrm{keV}$ interval. The gray scale represents counts increasing logarithmically from 0 (white) to 488 (black).

significantly smaller than the uncertainty in the absolute astrometry of Chandra. ${ }^{13}$

The sub-arcsecond angular resolution afforded by Chandra allows us to look for nebuar X-ray radiation surrounding the pulsar. Redback systems are of particular interest in this regard since any information about recent accretion could, in principle, be encoded in any anomalies in the diffuse X-rays associated with the pulsar. However, for $\gtrsim 2^{\prime \prime}$ away from PSR J1723-2837, there is no indication for excess emission in any direction away from the pulsar due to a bow shock or wind nebula. To formally verify this, we generated 20 simulated observations of PSR J1723-2837 with ChaRT ${ }^{14}$ and MARX 4.5, ${ }^{15}$ using the specifics of the Chandra observation and the best-fit X-ray spectrum as input. The average of the simulated point spread functions was subtracted from the observed image to identify any residual emission relative to the background level. For $\lesssim 1^{\prime \prime}$ from the center, the difference image reveals appreciable residuals (both positive and negative) that are azimuthally asymmetric. In principle, a shift between the position reported by wavdetect and the true source position could produce such residuals. To investigate this possibility, we have repeated the analysis using a range of shifts along the azimuthal direction in which the residuals are most significant in an attempt to minimize them. None yielded an improvement in the difference image. Based on this, the most likely explanation is that these residuals arise due to imperfections in the model of the High-Resolution Mirror Assembly optics (Juda \& Karovska 2010). ${ }^{16}$ Beyond $\sim 1^{\prime \prime}$ of the pulsar the difference image does not show any deviations from what is expected from a point source (Figure 5), confirming the absence of any diffuse emission surrounding the pulsar.

\footnotetext{
13 See http://cxc.harvard.edu/cal/ASPECT/celmon/.

14 The Chandra Ray Tracer, available at http://cxc.harvard.edu/soft/ ChaRT/cgi-bin/www-saosac.cgi

15 Available at http://space.mit.edu/cxc/marx/index.html.

16 See also http://hea-www.harvard.edu/ juda/memos/HEAD2010/ HEAD2010_poster.html and http://cxc.harvard.edu/cal/Hrc/PSF/acis_psf_ 2010oct.html
} 


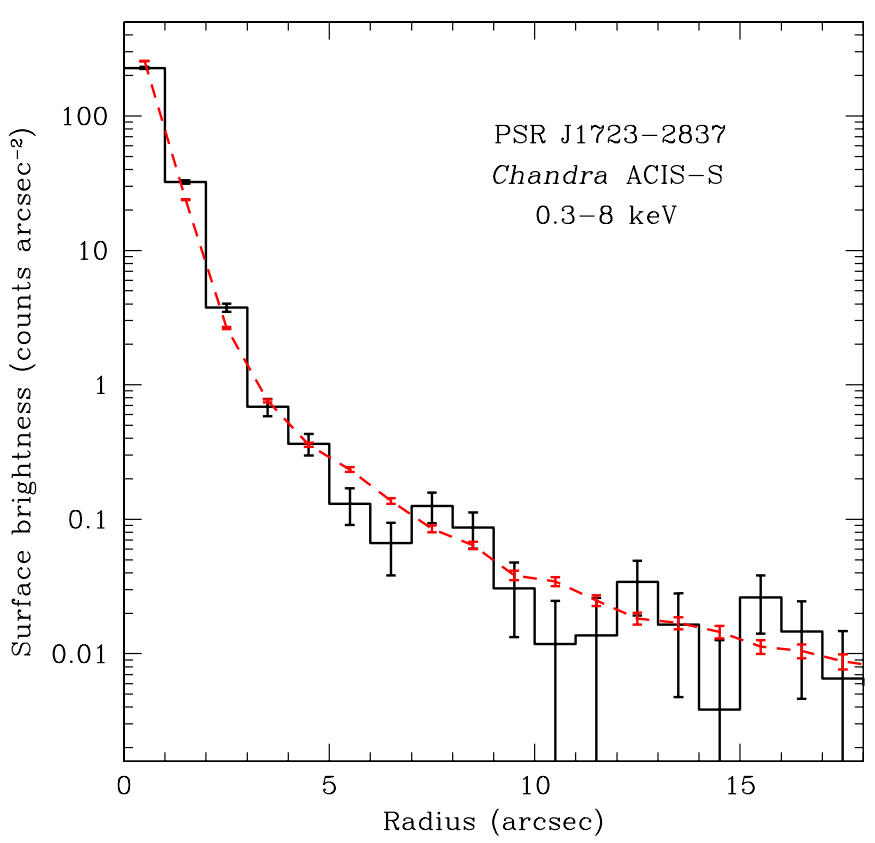

Figure 5. Radial profile of the observed ACIS-S point-spread function (PSF) of the X-ray counterpart of PSR J1723-2837 after background subtraction (histogram) and the mean of 20 synthetic PSFs (red dashed line).

(A color version of this figure is available in the online journal.)

Based on the background count rate around the pulsar $\left(2 \times 10^{-6}\right.$ counts s $^{-1} \operatorname{arcsec}^{-2}$ for $\left.0.3-6 \mathrm{keV}\right)$ and taking a typical power-law spectrum for X-ray pulsar wind nebulae (PWNe; Kargaltsev \& Pavlov 2008) with $\Gamma=1.5$, the limit on the PWN luminosity is $\sim 1 \times 10^{29} \mathrm{erg} \mathrm{s}^{-1}$ for a distance of $750 \mathrm{pc}$.

\section{PROBING THE PHYSICS OF THE INTRABINARY SHOCK}

In Bogdanov et al. (2011a), simple geometric modeling of PSR J1023+0038 revealed that an intrabinary shock localized primarily near the inner Lagrangian point and/or at the face of the companion can naturally account for the X-ray variability. In this interpretation, the obstruction of the observer's view of the X-ray region by the secondary causes the apparent decline in flux at $\phi \approx 0.25$. Given the qualitative similarities in observed $\mathrm{X}$-ray properties, it is highly probable that the location of the shock in J1723-2837 is the same.

Using this information it is possible to gain quantitative insight into the properties of the intrabinary shock. Due the compactness of the binary $\left(a \approx 2 \times 10^{11} \mathrm{~cm}\right)$, it is likely that the shock is formed in a relatively intense magnetic field, meaning that synchrotron produced by accelerated particles is the most probable X-ray emission mechanism. The resulting synchrotron luminosity is a function of the strength of the post-shock field and the ratio between the Poynting flux and particle flux $\sigma$ (the wind magnetization factor). If the wind is dominated by kinetic energy, $\sigma \approx 0.003$ (like in the Crab nebula; Kennel \& Coroniti 1984), while for a magnetically dominated wind, $\sigma \gg 1$. Based on the prescription presented by Arons \& Tavani (1993), the field strength immediately past the shock is defined by $B_{1}=[\sigma /(1+\sigma)]^{1 / 2}\left(\dot{E} / c f_{p} r^{2}\right)^{1 / 2}$, where $f_{p}$ defines the portion of the sky into which the pulsar wind is emitted, while $r$ is the separation between the pulsar and the shock front. For PSR J1723-2837, the approximate distance from the MSP to $L_{1}$ assuming $M_{\mathrm{MSP}}=1.4 M_{\odot}$ and $i=37^{\circ}$ is $r \approx 2 \times 10^{11} \mathrm{~cm}$. For a pulsar wind that is emitted isotropically $\left(f_{p}=1\right)$ with
$\dot{E}=4.6 \times 10^{34} \mathrm{erg} \mathrm{s}^{-1}$ this produces $B_{1} \approx 0.68 \mathrm{G}(\sigma=0.003)$ and $B_{1} \approx 12 \mathrm{G}(\sigma \gg 1)$. The resulting magnetic field strength past the shock is $B_{2}=3 B_{1} \sim 2 \mathrm{G}$ or $B_{2} \sim 37 \mathrm{G}$, respectively. In order to produce photons with energies $\varepsilon_{\mathrm{keV}}=0.3-8 \sim 1 \mathrm{keV}$ by synchrotron radiation, relativistic $e^{p m}$ with Lorentz factors $\gamma=2.4 \times 10^{5}\left(\varepsilon / B_{2}\right)^{1 / 2}$ (with $\varepsilon$ in units of $\mathrm{keV}$ and $B_{2}$ in $\mathrm{G})$ are required, which yields $\sim 0.2 \times 10^{5}(\sigma \gg 1)$ and $\sim 1 \times 10^{5}(\sigma=0.003)$. The associated radiative loss time is then $t_{\text {synch }}=5.1 \times 10^{8}\left(\gamma B_{2}^{2}\right)^{-1} \sim 2-145 \mathrm{~s}$ (Rybicki \& Lightman 1979). Assuming a shock region that is $\sim 1 R_{\odot}$, the radius of the companion's Roche lobe (Crawford et al. 2013), the residence times of the synchrotron-emitting $\mathrm{e}^{\mp}$ in the shock are $t_{\text {flow }}=c / 3 R \approx 13 \mathrm{~s}$ (for $\sigma=0.003$ ) and $t_{\text {flow }}=c / R \approx 40 \mathrm{~s}$ (for $\sigma \gg 1$ ).

The luminosity from the shock due to synchrotron radiation can be computed approximately using the expression $f_{\text {shock }} f_{\varepsilon} L_{\varepsilon}=f_{\text {synch }} f_{\gamma} f_{\text {geom }} \dot{E}$. Here $f_{\text {synch }}$ represents the radiative efficiency of the synchrotron mechanism, $f_{\gamma}$ corresponds to the portion of the wind power that goes into accelerating $e^{\mp}$ with Lorentz factor $\gamma, f_{\text {geom }}$ is the portion of the MSP outflow that encounters material from the secondary star, $f_{\text {shock }}$ is the fraction of the total system luminosity that arises due the shock, and $L_{\varepsilon}$ is the X-ray luminosity in the energy interval under consideration, while $f \varepsilon$ is the fraction of the total synchrotron spectrum falling in the observed energy band. Using $f_{\text {synch }}=\left(1+t_{\text {synch }} / t_{\text {flow }}\right)^{-1}$, we find values of $f_{\text {synch }} \approx 0.02$ and $f_{\text {synch }} \approx 0.8$, corresponding to $\sigma=0.003$ and $\sigma \gg 1$, respectively. For a radius of $\sim 1 R_{\odot}$, the secondary encounters $f_{\text {geom }} \approx 0.01$ of the pulsar's outflow if the MSP wind is uniformly emitted in all direction. However, it is highly probable that the MSP wind is significantly anisotropic, with most of it flowing out equatorially since such a geometry is observed in the Crab pulsar (Hester et al. 1995; Michel 1994). Given that during the LMXB accretion phase the pulsar spin axis vector has become parallel with the orbital angular momentum vector, the wind should be emitted predominantly in the orbital plane (Bhattacharya \& van den Heuvel 1991). For a wind emitted only in the orbital plane, the companion star would intercept $f_{\text {geom }} \approx 0.08$ of the total wind energy. Thus, we set $0.01<f_{\text {geom }}<0.08$.

Based on the phase-resolved spectroscopic analysis, the intrinsic shock luminosity (in the absence of eclipses) can be estimated to be $L_{\varepsilon} \approx 1 \times 10^{32} \mathrm{erg} \mathrm{s}^{-1}$ for particles with $\gamma \approx 10^{5}$. With $f_{\text {shock }} \approx 1$ as obtained from the spectroscopic analysis, we find $27 \lesssim f_{\gamma} \lesssim 218(\sigma=0.003)$ and $0.14 \lesssim f_{\gamma} \lesssim 1.1$ $(\sigma \gg 1)$. In the case of $\sigma=0.003$, the implied range of $f_{\gamma}$ is clearly unphysical as it exceeds unity, even if the shock region receives $100 \%$ of the pulsar wind power. This problem is further exacerbated if the cutoff of the energy spectrum is significantly above $10 \mathrm{keV}$, such that the observed flux is only a fraction of the emitted flux. This would increase the fraction of the wind power that has to go in electron acceleration. This implies that for PSR J1723-2837 the wind in the vicinity of the shock is probably magnetically dominated.

In the case of $\sigma \gg 1$, obtaining comparable values to that of the Crab pulsar $\left(f_{\gamma}=0.04\right)$ can be obtained by assuming that the bulk of the X-ray-emitting region is confined to an equatorial strip (as illustrated in Figure 6). It is interesting to note that a similar feature is necessary to account for the peculiar He $\mathrm{I}$ lines seen in the analogous MSP binary PSR J1740-5340 in NGC 6397 (Ferraro et al. 2003). This requires either a sheet-like pulsar wind and/or a strong outflow from the companion that is preferentially emitted along the stellar equator. Alternatively, a realistic value of $f_{\gamma}$ can be achieved for both scenarios 


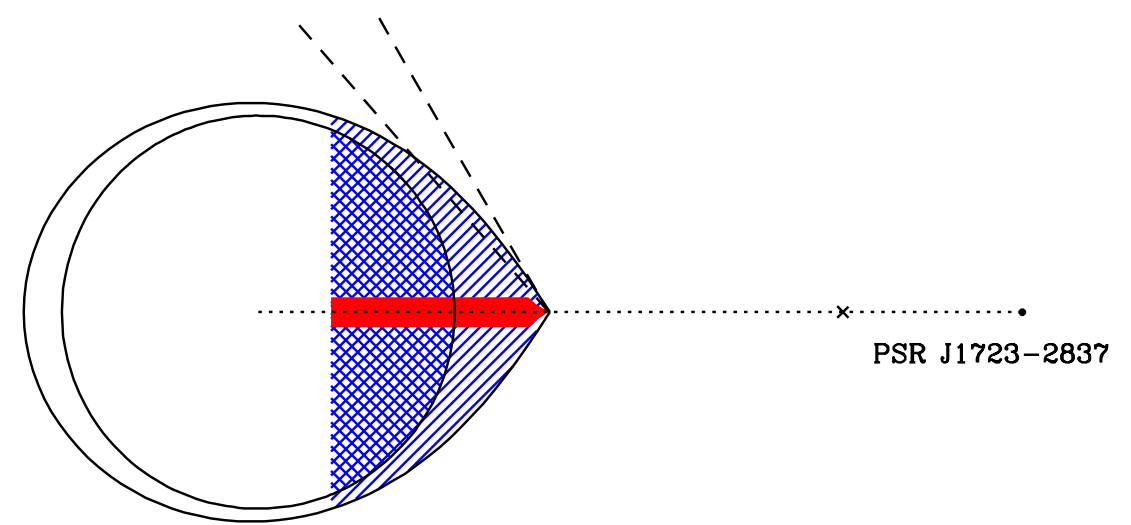

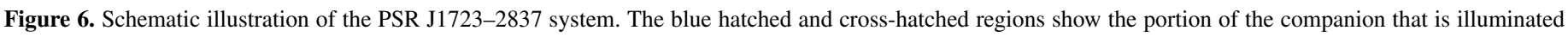

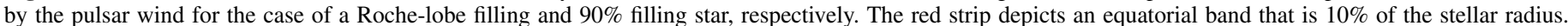

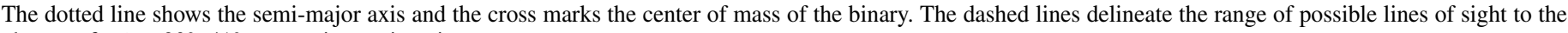
observer for $i=30^{\circ}-41^{\circ}$ at superior conjunction.

(A color version of this figure is available in the online journal.)

if the secondary star has a relatively high surface magnetic field, $\sim 10^{2-3} \mathrm{G}$ (see Donati \& Landstreet 2009, and references therein), at the high end of fields measured for main-sequence stars. However, for values $\lesssim 10^{2} \mathrm{G}$, the emission region still needs to be a factor of $\lesssim 10$ smaller than the secondary star.

In Crawford et al. (2013), it was suggested that the measured $\dot{P}$ and hence all parameters derived from it are affected and possibly dominated by the kinematic (Shklovskii) effect. This implies that the pulsar's true spin-down luminosity is significantly smaller than the derived value of $\dot{E}=4.6 \times 10^{34} \mathrm{erg} \mathrm{s}^{-1}$. If we consider a smaller intrinsic $\dot{E}$ in the calculations above, the required X-ray conversion efficiency becomes unrealistically large, unless we invoke a very compact X-ray-emitting shock region and/or a companion star with a $\sim 10^{3} \mathrm{G}$ surface magnetic field. Thus, in order to further constrain the physics and geometry of the intrabinary shock it is important to determine the intrinsic $\dot{P}$ of the pulsar by way of continuing radio timing observations or optical proper motion measurements.

\section{FERMI LAT ANALYSIS}

\subsection{Binned Likelihood Analysis}

PSR J1723-2837 does not fall within the 95\% confidence region of any source in the Fermi-LAT two-year point source catalog (Nolan et al. 2012). The two nearest published sources, 2FGL J1717.3-2809 and 2FGL J1728.0-2737c, both lie 1.4 away. A visual inspection of the nearly five years of Fermi-LAT data we have retrieved reveals no obvious $\gamma$-ray source at the pulsar position. This is in large part due the pulsar being situated only $4^{\circ}$ above the Galactic plane, where the diffuse Galactic emission component is quite strong and source confusion can be particularly problematic.

To formally establish whether PSR J1723-2837 is a $\gamma$-ray source, we carried out a binned likelihood analysis by considering a source at the position of the pulsar modeled by a power-law with an exponential cutoff, with the form $d N / d t \propto E^{-\Gamma} \exp \left(-E / E_{c}\right)$, where $\Gamma$ is the spectral photon index and $E_{c}$ is the spectrum cutoff energy. The parameters of the putative $\gamma$-ray pulsar, the 44 sources within $10^{\circ}$ of the pulsar, and the normalization factors of diffuse components were left free in the fit. We also consider emission from sources up to $15^{\circ}$ away but keep their parameters fixed. The source model also included contributions from the extragalactic diffuse emission and the residual instrumental background, jointly modeled using the iso_p7v6source template, and from the Galactic diffuse emission, modeled with the gal_2yearp7v6_v0 map cube.

The likelihood analysis yields a source test statistic (TS, see Nolan et al. 2012, for a definition) value of 57, corresponding to $\mathrm{a} \sim 7.6 \sigma$ significance, for the putative pulsar $\gamma$-ray counterpart. However, the best-fit pulsar spectrum tends toward very steep photon indices $(\Gamma \sim 3)$ and low values of the cutoff energy $\left(E_{c} \lesssim 500 \mathrm{MeV}\right)$, likely owing to the paucity of photons above $\sim 500 \mathrm{MeV}$. These values are substantially different from those of the current sample of $\gamma$-ray detected MSPs (see Table 10 in Abdo et al. 2013), which have average photon index and cutoff energy of $\Gamma=1.3$ and $E_{c}=2.2 \mathrm{GeV}$, with the lowest values being $\Gamma=1.9$ and $E_{c}=1.1 \mathrm{GeV}$. Fixing the pulsar parameters to the latter set of values results in $\mathrm{TS}=45(\sim 6.7 \sigma)$. To mitigate the effect of the overwhelming Galactic diffuse background at $\sim 100 \mathrm{MeV}$, we also carried out the likelihood analysis for photon energies $>300 \mathrm{MeV}$. The result is a substantially diminished significance of the source (with TS $=22$, corresponding to $\sim 4.7 \sigma$ ) and a best fit with a very steep power-law $\Gamma \approx 3$ and an abnormally low cutoff $E_{c} \approx 200-500 \mathrm{MeV}$. Using $\Gamma=1.9$ and $E_{c}=1.1 \mathrm{GeV}$ as fixed parameters results in TS $=20$.

As the "c" suffix designates, the source 2FGL J1728.0-2737c is confused, indicating that its reported source position and spectrum are unreliable. Based on this, we consider the possibility that this $\gamma$-ray source is perhaps the pulsar and remove it from the input model. The result is TS $=74.4(\sim 8.6 \sigma)$ for the pulsar for $>100 \mathrm{MeV}$ but again with a very steep spectrum and a low cutoff energy for the best fit. In this case, fixing the parameters to $\Gamma=1.9$ and $E_{c}=1.1 \mathrm{GeV}$, results in $\mathrm{TS}=65.3$, corresponding to $\sim 8 \sigma$. However, the same fit for $>300 \mathrm{MeV}$ results only in TS $=12.7$.

Although from a statistical standpoint the pulsar is consistent with being a $\gamma$-ray source, the veracity of the $\gamma$-ray detection appears to depend strongly on the accuracy of the Galactic diffuse model. The tendency toward abnormal values of $\Gamma$ and $E_{c}$, as well as the dramatic decline in the source significance for $>300 \mathrm{MeV}$, is an indication that the bulk of $\gamma$-ray emission at the pulsar position may not actually be associated with PSR J1723-2837 and is possibly due to excess diffuse emission that is not properly accounted for in the current model. If the $\gamma$-ray emission is in fact associated with the pulsar, the implied energy flux from PSR J1723-2837 assuming $\Gamma=1.9$ and $E_{c}=1.1 \mathrm{GeV}$ is $F_{\gamma} \approx 2 \times 10^{-11} \mathrm{erg} \mathrm{cm}^{-2} \mathrm{~s}^{-1}$ for energies 
above $100 \mathrm{MeV}$. For $D=750 \mathrm{pc}$, this corresponds to a $\gamma$-ray luminosity of $L_{\gamma} \approx 2 \times 10^{33} \mathrm{erg} \mathrm{s}^{-1}$. Given the ambiguity regarding the pulsar detection, this provides a conservative upper limit on the $\gamma$-ray production efficiency of $\sim 5 \%$ of the pulsar spin-down luminosity, toward the low end of values in the current sample of Fermi-LAT MSPs (see Table 10 in Abdo et al. 2013). This could mean that PSR J1723-2837 is either one of the least efficient $\gamma$-ray-emitting MSPs or, if the detection is spurious, the $\gamma$-ray emission pattern is not directed toward us (Romani \& Shaw 2011).

\subsection{Photon-weighted Pulsation Search}

As reported in (Crawford et al. 2013), the timing irregularities associated with this PSR J1723-2837 requires the addition of multiple orbital period derivatives in order to obtain a satisfactory radio timing solution. As a result, the best radio timing ephemeris cannot be reliably extrapolated to fold FermiLAT photons over the entire $\sim 5 \mathrm{yr}$ span of the mission. As a result, we restrict our analysis to events detected in the interval over which the pulsar ephemeris is valid (MJDs 55101.8-55803.8).

Folding the Fermi-LAT photons extracted with various energy cuts and acceptance cone radii does not yield statistically significant $\gamma$-ray pulsations. However, as demonstrated in recent studies (Kerr 2011; Guillemot et al. 2012) weighting the $\gamma$-ray photons by the probability that they originate from a pulsar significantly enhances the sensitivity to faint pulsations. This is especially critical for PSR J1723-2837 owing to its proximity to the Galactic plane $(b=4.2)$, where the diffuse $\gamma$-ray background is strong. The resulting best-fit source model from the binned likelihood analysis was used in conjunction with the gtsrcprob script to assign a probability to each photon of being associated with PSR J1723-2837. Since there are two extended source within $10^{\circ}$ of the pulsar (W28 and W30), the gtdiffrsp was first used to compute the diffuse response over this region.

Based on this, we folded only photons with probabilities greater than 0.05 using the Fermi plug-in ${ }^{17}$ for the tempo $2^{18}$ pulsar timing package and the best available radio ephemeris (Crawford et al. 2013). The photon weights were calculated by using the best-fit spectral model of the region around the $\gamma$-ray source. Folding the extracted probability-weighted Fermi-LAT photons with energies $>100 \mathrm{MeV}$ with the ephemeris of PSR J1723-2837 yields no statistically significant pulsations. Due to the overwhealming background at low energies a range of energy bands was also considered, but still no pulsations were detected.

In principle, at least a portion of the $\gamma$-ray emission associated with PSR J1723-2837 could arise from the same intrabinary shock that produces the non-thermal X-ray emission. This scenario has been proposed by Tam et al. (2010) to explain the $\gamma$-ray emission associated with PSR J1023+0038. In fact, a similarly steep photon index to that found in Section 7.1 is derived for PSR J1023+0038 (2.9 \pm 0.2$)$, suggesting that for PSR J1723-2837 the same $\gamma$-ray production mechanism dominates. This would make these two pulsars exceptional among the "redbacks" in the field of the Galaxy, which tend to exhibit strong pulsation at the spin period (Ray et al. 2012). To investigate this possibility, we folded the data at the binary period but found no statistically significant variability.

\footnotetext{
17 See http://fermi.gsfc.nasa.gov/ssc/data/analysis/user/Fermi_plug_doc.pdf.

$18 \mathrm{http}: / /$ sourceforge.net/projects/tempo2/
}

\section{CONCLUSION}

We have presented an analysis of XMM-Newton and Chandra observations of the nearby PSR J1723-2837 "redback" MSP system. The X-ray spectrum show a strong non-thermal component that accounts for most if not all of the emission in the $0.3-8 \mathrm{keV}$ band, which exhibits large-amplitude variability as a function of the binary orbital period. This pronounced flux modulation, with a significant decline in flux at conjunction, appears to be one of the defining characteristics of so-called redback systems. As such, it can serve as a convenient identifier of additional members of this population, especially in instances where radio detection is difficult due to prolonged eclipses. For instance, strong outflows in similar systems may inhibit detection of radio pulsations, rendering them perpetually eclipsed (Tavani 1991). Such an occurrence is believed to be the cause of the recent disappearance of PSR J1023+0038 at radio frequencies (Stappers et al. 2013).

There is no indication of an X-ray wind nebula associated with the pulsar. The lack of a discernable PWN down to a limit of $\lesssim 3.6 \times 10^{29} \mathrm{erg} \mathrm{s}^{-1}$, corresponding to $\lesssim 7 \times 10^{-6}$ of the pulsar's $\dot{E}$, indicates that the combination of the low density of the surrounding interstellar medium, unfavorable wind geometry, and/or possibly low space velocity are likely not conducive to the production of an X-ray-bright bow shock. Thus, X-ray bow shocks associated with nearby MSPs remain quite rare, with only two objects, PSRs B1957+21 (Stappers et al. 2003) and J2124-3358 (Hui \& Becker 2006), exhibiting prominent bow shock emission.

A likelihood analysis of the Fermi-LAT emission in the vicinity of PSR J1723-2837 reveals that the pulsar is consistent with being a $\gamma$-ray point source although owing to the strong diffuse background a detection cannot be established conclusively. There are no statistically significant $\gamma$-ray pulsations detected even using photon probability weights. The absence of pulsed emission could arise due to one or more of the following reasons: (1) the $\gamma$-ray emission at the pulsar position is unrelated to the pulsar indicating that PSR J1723-2837 is sub-luminous in $\gamma$-rays or its $\gamma$-ray emission pattern is not favorably oriented; (2) the pulse shape is not favorable (e.g., due to a high duty cycle), which combined with the high background and the paucity of source photons above $300 \mathrm{MeV}$ results in a non-detection. There is also no evidence for orbital-phase-dependent variability if the $\gamma$-rays.

By analogy with PSR J1824-2452I, the redback and bona fide X-ray binary/radio MSP transition system (Papitto et al. 2013), PSR J1723-2837 could also experience a switch to an accretion disk state. Therefore, as the nearest such system, PSR J1723-2837 warrants close scrutiny at all wavelengths as it provides the best-suited target for studying the transition process of MSPs from accretion to rotation power (and vice versa) and the circumstances surrounding it.

This work was funded in part by NASA Chandra grants GO2-13049A/B awarded through Columbia University and West Virginia University and issued by the Chandra X-ray Observatory Center, which is operated by the Smithsonian Astrophysical Observatory for and on behalf of NASA under contract NAS8-03060. A portion of the results presented was based on observations obtained with XMM-Newton, an ESA science mission with instruments and contributions directly funded by ESA Member States and NASA. This research has made use of the NASA Astrophysics Data System (ADS) and 
software provided by the Chandra X-ray Center (CXC) in the application package CIAO. We acknowledge the use of data and software facilities from the FSSC, managed by the HEASARC at the Goddard Space Flight Center.

Facilities: XMM, CXO, Fermi

\section{REFERENCES}

Abdo, A. A., Ajello, M., Allafort, A., et al. 2013, ApJS, 208, 17

Anders, E., \& Grevesse, N. 1989, GeCoA, 53, 197

Archibald, A. M., Kaspi, V. M., Bogdanov, S., et al. 2010, ApJ, 722, 88

Archibald, A. M., Stairs, I. H., Ransom, S. M., et al. 2009, Sci, 324, 1411

Arons, J., \& Tavani, M. 1993, ApJ, 403, 249

Bhattacharya, D., \& van den Heuvel, E. P. J. 1991, PhR, 203, 1

Bogdanov, S. 2013, ApJ, 762, 96

Bogdanov, S., Archibald, A. M., Hessels, J. W. T., et al. 2011a, ApJ, 742, 97

Bogdanov, S., \& Grindlay, J. E. 2009, ApJ, 703, 1557

Bogdanov, S., Grindlay, J. E., Heinke, C. O., et al. 2006, ApJ, 646, 1104

Bogdanov, S., Grindlay, J. E., \& van den Berg, M. 2005, ApJ, 630, 1029

Bogdanov, S., Rybicki, G. B., \& Grindlay, J. E. 2007, ApJ, 670, 668

Bogdanov, S., van den Berg, M., Heinke, C. O., et al. 2010, ApJ, 709, 241

Bogdanov, S., van den Berg, M., Heinke, C. O., et al. 2011b, ApJ, 730, 81

Cameron, P. B., Rutledge, R. E., Camilo, F., et al. 2007, ApJ, 660, 587

Camilo, F., Lorimer, D. R., Freire, P., Lyne, A. G., \& Manchester, R. N. 2000, ApJ, 535, 975

Cordes, J. M., \& Lazio, T. J. W. 2002, arXiv:astro-ph/0207156

Crawford, F., Lyne, A. G., Stairs, I. H., et al. 2013, ApJ, 776, 20

D’Amico, N., Lyne, A. G., Manchester, R. N., Possenti, A., \& Camilo, F. 2001, ApJL, 548, L171

Davis, J. E. 2001, ApJ, 562, 575

Deller, A. T., Archibald, A. M., Brisken, W. F., et al. 2012, ApJL, 756, L25

De Luca, A., \& Molendi, S. 2004, A\&A, 419, 837

Donati, J.-F., \& Landstreet, J. D. 2009, ARA\&A, 47, 333

Faulkner, A. J., Stairs, I. H., Kramer, M., et al. 2004, MNRAS, 355, 147

Ferraro, F. R., Sabbi, E., Gratton, R., et al. 2003, ApJ, 584, L13

Freire, P. C., Camilo, F., Kramer, M., et al. 2003, MNRAS, 340, 1359

Fruchter, A. S., Stinebring, D. R., \& Taylor, J. H. 1988, Natur, 333, 237

Fruscione, A., McDowell, J. C., Allen, G. E., et al. 2006, Proc. SPIE, 6270, $62701 \mathrm{~V}$

Gentile, P., Roberts, M., McLaughlin, M., et al. 2013, ApJ, submitted (eprint arXiv:1305.6799)
Guillemot, L., Johnson, T. J., Venter, C., et al. 2012, ApJ, 744, 33

Hessels, J. W. T., Roberts, M. S. E., McLaughlin, M. A., et al. 2011, in AIP Conf. Ser. 1357, Radio Pulsars: An Astrophysical Key to Unlock the Secrets of the Universe, ed. M. Burgay et al. (Melville, NY: AIP), 40

Hester, J. J., Scowen, P. A., Sankrit, R., et al. 1995, ApJ, 448, 240

Hui, C. Y., \& Becker, W. 2006, A\&A, 448, L13

Juda, M., \& Karovska, M. 2010, BAAS, 41, 722

Kalberla, P. M. W., Burton, W. B., Hartmann, D., et al. 2005, A\&A, 440,775

Kaplan, D. L., Stovall, K., Ransom, S. M., et al. 2012, ApJ, 753, 174

Kargaltsev, O., \& Pavlov, G. G. 2008, in AIP Conf. Proc. 983, 40 Years of Pulsars: Millisecond Pulsars, Magnetars and More, ed. C. Bassa et al. (Melville, NY: AIP), 171

Kennel, C. F., \& Coroniti, F. V. 1984, ApJ, 283, 694

Kerr, M. 2011, ApJ, 732, 38

Liedahl, D. A., Osterheld, A. L., \& Goldstein, W. H. 1995, ApJL, 438, L115

Mewe, R., Gronenschild, E. H. B. M., \& van den Oord, G. H. J. 1985, A\&AS, 62, 197

Mewe, R., Lemen, J. R., \& van den Oord, G. H. J. 1986, A\&AS, 65, 511

Michel, F. C. 1994, ApJ, 431, 397

Nolan, P. L., Abdo, A. A., Ackermann, M., et al. 2012, ApJS, 199, 31

Paltani, S. 2004, A\&A, 420, 789

Papitto, A., Ferrigno, C., Bozzo, E., et al. 2013, Natur, 501, 517

Patruno, A., Archibald, A. M., Hessels, J. W. T., et al. 2013, ApJL, in press (arXiv: 1310.7549)

Pletsch, H. J., Guillemot, L., Allen, B., et al. 2012, ApJ, 744, 105

Ray, P. S., Abdo, A. A., Parent, D., et al. 2012, 2011 Fermi Symposium Proceedings, eConf C110509 (arXiv: 1205.3089)

Romani, R. W., \& Shaw, M. S. 2011, ApJL, 743, L26

Rybicki, G. B., \& Lightman, A. P. 1979, Radiative Processes in Astrophysics (New York: Wiley)

Stappers, B. W., Archibald, A., Bassa, C., et al. 2013, ATel, 5031, 1

Stappers, B. W., Gaensler, B. M., Kaspi, V. M., van der Klis, M., \& Lewin, W. H. G. 2003, Sci, 299, 1372

Strüder, L., Briel, U., Dennerl, K., et al. 2001, A\&A, 365, L18

Tam, P. H. T., Hui, C. Y., Huang, R. H. H., Kong, A. K. H., et al. 2010, ApJ, 724, 207

Tavani, M. 1991, ApJL, 379, L69

Turner, M. J. L. 2001, A\&A, 365, L27

Zavlin, V. E. 2006, ApJ, 638, 951

Zavlin, V. E., \& Pavlov, G. G. 1998, A\&A, 329, 583

Zavlin, V. E., Pavlov, G. G., \& Shibanov, Yu. A. 1996, A\&A, 315, 141 